\title{
Quantitative Diagnostic Performance of Myocardial Perfusion SPECT with Attenuation Correction in Women
}

\author{
Arik Wolak ${ }^{1,2}$, Piotr J. Slomka ${ }^{1-3}$, Mathews B. Fish ${ }^{4}$, Santiago Lorenzo ${ }^{5}$, Daniel S. Berman ${ }^{1-3}$, and Guido Germano ${ }^{1-3}$ \\ ${ }^{I}$ Department of Imaging, Cedars-Sinai Medical Center, Los Angeles, California; ${ }^{2}$ Department of Medicine, Cedars-Sinai Medical \\ Center, Los Angeles, California; ${ }^{3}$ Department of Medicine, David Geffen School of Medicine at UCLA, Los Angeles, California; \\ ${ }^{4}$ Oregon Heart and Vascular Institute, Sacred Heart Medical Center, Eugene, Oregon; and ${ }^{5}$ Department of Human Physiology, \\ University of Oregon, Eugene, Oregon
}

Attenuation correction (AC) for myocardial perfusion SPECT (MPS) had not been evaluated separately in women despite specific considerations in this group because of breast photon attenuation. We aimed to evaluate the performance of $A C$ in women by using automated quantitative analysis of MPS to avoid any bias. Methods: Consecutive female patients - 134 with a low likelihood (LLk) of coronary artery disease (CAD) and 114 with coronary angiography performed within less than 3 mo of MPS - who were referred for rest-stress electrocardiographygated ${ }^{99 m}$ Tc-sestamibi MPS with AC were considered. Imaging data were evaluated for contour quality control. An additional 50 LLk studies in women were used to create equivalent normal limits for studies with $A C$ and with no correction (NC). An experienced technologist unaware of the angiography and other results performed the contour quality control. All other processing was performed in a fully automated manner. Quantitative analysis was performed with the Cedars-Sinai myocardial perfusion analysis package. All automated segmental analyses were performed with the 17-segment, 5-point American Heart Association model. Summed stress scores (SSS) of $\geq 3$ were considered abnormal. Results: CAD ( $\geq 70 \%$ stenosis) was present in 69 of 114 patients (60\%). The normalcy rates were $93 \%$ for both NC and AC studies. The SSS for patients with CAD and without CAD for NC versus $A C$ were $10.0 \pm 9.0$ (mean $\pm S D$ ) versus $10.2 \pm 8.5$ and $1.6 \pm$ 2.3 versus $1.8 \pm 2.5$, respectively; $P$ was not significant (NS) for all comparisons of NC versus AC. The SSS for LLk patients for $\mathrm{NC}$ versus $\mathrm{AC}$ were $0.51 \pm 1.0$ versus $0.6 \pm 1.1$, respectively; $P$ was NS. The specificity for both NC and AC was $73 \%$. The sensitivities for $\mathrm{NC}$ and $\mathrm{AC}$ were $80 \%$ and $81 \%$, respectively, and the accuracies for NC and AC were $77 \%$ and $78 \%$, respectively; $P$ was NS for both comparisons. Conclusion: There are no significant diagnostic differences between automated quantitative MPS analyses performed in studies processed with and without $\mathrm{AC}$ in women.

Received Nov. 23, 2007; revision accepted Feb. 18, 2008.

For correspondence or reprints contact: Piotr J. Slomka, Cedars-Sinai Medical Center, Room A047, 8700 Beverly Blvd., Los Angeles, CA 90048.

E-mail: slomkap@cshs.org

COPYRIGHT @ 2008 by the Society of Nuclear Medicine, Inc.
Key Words: attenuation correction; myocardial perfusion; quantification; women; gender

J Nucl Med 2008; 49:915-922

DOI: 10.2967/jnumed.107.049387

O the diagnostic accuracy (through higher specificity) for the detection of coronary artery disease (CAD) when myocardial perfusion SPECT (MPS) is attenuation corrected (1-7). These studies were based on various implementations of different attenuation correction (AC) hardware designs, and most of them relied primarily on visual interpretation which, in this context, is often associated with bias when improved technology is being evaluated (7). MPS can be automatically quantified for objective comparison of images with $\mathrm{AC}$ and with no correction (NC), yet only a few published studies have addressed the diagnostic accuracy of AC in such a quantitative manner without any visual interpretation of $\mathrm{AC}$ and NC data (8-12).

Of interest is that none of the quantitative studies and only one older visual study (2) reported quantitative attenuationcorrected data specifically in women, even though attenuation artifacts in women are known to be of a different nature from those in men (13). Therefore, we aimed to evaluate the performance of AC in women by using automated quantitative analysis of MPS to avoid any possible bias associated with visual interpretation.

\section{MATERIALS AND METHODS}

\section{Patients}

The subjects for this study were selected consecutively from 2,624 female patients who were referred to the Nuclear Medicine Department, Sacred Heart Medical Center, Eugene, OR, from March 1, 2003, to December 31, 2004, for rest-stress electrocardiography (ECG)-gated MPS and who met various criteria of this study, as described later. During this period, all studies were performed with a rest sestamibi-stress sestamibi protocol for the 
simultaneous acquisition of AC MPS and NC MPS studies, as detailed later. The retrospective use of clinical data in this study was approved by the Institutional Review Board at Sacred Heart Medical Center. Clinical and imaging data with all personal identifiers removed were transmitted to Cedars-Sinai Medical Center for analysis.

Low-Likelihood (LLk) Group. LLk of CAD $(<5 \%)$ was defined on the basis of age, sex, pretest symptoms, and ECG response to treadmill stress testing (14). Accordingly, LLk patients were selected only from 203 patients who had treadmill stress testing and reached an adequate level of treadmill stress ( $\geq 85 \%$ of the predicted maximum heart rate). These patients had no history of CAD (prior myocardial infarction or coronary revascularization) or other confounding cardiac conditions, including congestive heart failure, cardiomyopathy, significant valvular or congenital heart disease, left bundle branch block, or paced rhythm. These patients did not have coronary angiography. Furthermore, these patients had to have MPS studies of good to excellent quality, normal ventricular volumes, wall motion, and global systolic function, and no evidence of transient ischemic dilatation, as judged by the director of the MPS laboratory at which the data were acquired. The final group for the evaluation of normalcy rates consisted of 134 consecutive female patients meeting these criteria.

Angiography Group. The group for the evaluation of diagnostic sensitivity, specificity, and accuracy for the detection of CAD consisted of 114 consecutive female patients who had MPS with an adequate stress test and coronary angiography within $60 \mathrm{~d}$ without an intervening clinical cardiac event. Of these, 90 (78\%) had adenosine stress. The 24 patients who had treadmill stress achieved a heart rate (mean \pm SD) of $88 \% \pm 5 \%$ of the predicted maximum heart rate for their ages at the time of injection of the radiotracer. The consecutive patients undergoing catheterization in this study excluded patients with previous revascularization, cardiomyopathy, valvular disease, left bundle branch block, or paced rhythm as well as 7 additional patients who had insufficient quality of MPS studies. The director of the MPS laboratory at which the data were acquired judged the image quality.

Normal Database Population. Normal limits were evaluated and created for AC and NC studies from a group of 50 additional female patients who were selected from 187 consecutive patients with LLk of CAD $(<5 \%)$, as described later. Additional criteria were applied to these 50 patients for the creation of the normal database. All had to demonstrate at most very slight overlapping extracardiac radioactivity and had to show visually normal reststress MPS images. Strict LLK criteria could only be determined for the exercise studies (ECG response); therefore, these studies were used to build the normal database. We previously showed, however, that exercise and adenosine stress can be interchanged and can be applied to a test population when adenosine stress or exercise is performed (15). The clinical characteristics for all of the patients in this study are shown in Table 1. With the Database Editor function for QPS (16), normal limits were created for the same group of patients.

\section{Rest and Stress Imaging Protocols}

Patient preparation included no caffeine-containing drinks, food, or medications for $24 \mathrm{~h}$ and no methyxanthine medications for $36-48 \mathrm{~h}$ unless clinically contraindicated. Patients were requested to be free of short-acting nitrates for $2 \mathrm{~h}$, long-acting nitrates for $6 \mathrm{~h}$, calcium channel blockers for $24 \mathrm{~h}$, and $\beta$-blockers for $48 \mathrm{~h}$ before the test. Studies were performed with ${ }^{99 \mathrm{~m}} \mathrm{Tc}$ rest
TABLE 1

Patient Characteristics

\begin{tabular}{|c|c|c|c|}
\hline Parameter & $\begin{array}{l}\text { Database } \\
\text { group } \\
(n=50)\end{array}$ & $\begin{array}{l}\text { LLk group } \\
(n=134)\end{array}$ & $\begin{array}{c}\text { Angiography } \\
\text { group } \\
(n=114)\end{array}$ \\
\hline Age $(y)$, mean $\pm S D$ & $56.7 \pm 14.7$ & $52.2 \pm 5.0$ & $65.2 \pm 21.2$ \\
\hline $\begin{array}{l}\mathrm{BMI}\left(\mathrm{kg} / \mathrm{m}^{2}\right) \\
\quad \text { mean } \pm \mathrm{SD}\end{array}$ & $30.5 \pm 6.2$ & $28.3 \pm 15.2$ & $30.7 \pm 6.0$ \\
\hline $\mathrm{BMI}$ of $\leq 30$ & $25(50)$ & $96(70)$ & $65(47)$ \\
\hline Diabetes & $8(16)$ & $12(9)$ & $35(30)$ \\
\hline Hypertension & $22(44)$ & $57(42)$ & $84(73)$ \\
\hline Hypercholesterolemia & $19(38)$ & $47(34)$ & $53(46)$ \\
\hline Chest pain & $12(24)$ & $54(39)$ & $85(74)$ \\
\hline Adenosine & $0(0)$ & $0(0)$ & $143(76)$ \\
\hline
\end{tabular}

Data are reported as number (percentage), unless otherwise indicated.

and ${ }^{99 m}$ Tc stress protocols. A same-day rest-stress protocol was used for women who weighed less than $90 \mathrm{~kg}(200 \mathrm{lb})$ or whose body mass index (BMI) was less than 35. A 2-d rest-stress or stress-rest protocol was used for women whose weight or BMI was above these levels. The weight- and BMI-related ${ }^{99 \mathrm{~m}} \mathrm{Tc}-$ sestamibi dose schedules were approximately 315-429 MBq for rest MPS and 1,092-1,554 MBq for stress MPS. For the 2-d protocols, the "stress" dose was used for both the rest and the stress portions of the study.

\section{Stress Testing}

Patients undergoing exercise stress performed a symptomlimited treadmill test with a standard Bruce protocol. At nearly maximum exercise, ${ }^{99} \mathrm{~m}$ Tc-sestamibi was injected intravenously. Exercise was continued at the maximum workload for 1.5-2.0 min, when possible. When exercise testing was contraindicated or unsuitable, a pharmacologic stress test was performed with an infusion of adenosine at $140 \mu \mathrm{g} \cdot \mathrm{kg}^{-1} \cdot \mathrm{min}^{-1}$ for $5 \mathrm{~min}$. At the end of the second minute, ${ }^{99 \mathrm{~m} T c-s e s t a m i b i}$ was injected. If able, during adenosine infusion, patients performed low-level treadmill exercise on a $0 \%$ grade at $0.8-1.7$ miles per hour.

\section{Image Acquisition}

Image acquisition was started at 60 min after the administration of ${ }^{99 \mathrm{~m} T c}$-sestamibi at rest or during adenosine infusion with the patient at rest and at 15-45 min after radiopharmaceutical injection during treadmill testing or adenosine infusion with low-level exercise. The patients drank $480 \mathrm{~mL}$ ( $16 \mathrm{oz})$ of water immediately before imaging. MPS images were acquired by use of Vertex dual-detector scintillation cameras with low-energy, high-resolution collimators, Vantage Pro AC hardware and software (Philips), and two ${ }^{153} \mathrm{Gd}$ scanning line sources, resulting in the simultaneous acquisition of ECG-gated emission and transmission images. These images were acquired over a $180^{\circ}$ noncircular orbit from $45^{\circ}$ right anterior oblique to left posterior oblique, with a $64 \times 64$ matrix (pixel size, $0.64 \mathrm{~cm}$ ) for emission images and a $128 \times 128$ matrix (pixel size, $0.32 \mathrm{~cm}$ ) for transmission images, and energy windows of $140 \mathrm{keV} \pm$ $20 \%$ for ${ }^{99 \mathrm{~m}} \mathrm{Tc}, 118 \mathrm{keV} \pm 12 \%$ for scatter, and $100 \mathrm{keV} \pm 20 \%$ for ${ }^{153} \mathrm{Gd}$. At each of the 64 projection angles, the image data were recorded into 8 equal ECG-gated time bins. Before imaging, 5-s 
transmission and scatter data were obtained over the patient's heart to determine an adequate time per projection allowing for a transmission count density resulting in a valid attenuation map. The times per projection used in this study were $45-50 \mathrm{~s}$ for rest MPS and $30-40 \mathrm{~s}$ for stress MPS.

\section{Tomographic Reconstruction}

Tomographic reconstruction was performed with AutoSpect and Vantage Pro programs (Philips Medical Systems). All emission images were automatically corrected for nonuniformity, radioactive decay, and motion during acquisition and were subjected to 3-point spatial smoothing. The mechanical center of rotation was determined to align the projection data to the reconstruction matrix. The NC stress MPS images were reconstructed by filtered backprojection with a Butterworth filter (order, 5; cutoff, 0.66).

Attenuation maps, corrected for ${ }^{99 \mathrm{~m}} \mathrm{Tc}$ downscatter, were reconstructed by use of a Bayesian prior approach after logarithmic inversion and normalization to a reference scan and the application of a Butterworth filter (order, 5; cutoff, 0.5). The attenuation maps were reconstructed with 12 iterations and a filtered backprojection initial estimate. With the attenuation maps and the emission data, the $\mathrm{AC}$ images were reconstructed by use of a maximum-likelihood algorithm with 30 iterations and a uniform initial estimate. Incorporated into this reconstruction was scatter correction into the emission photopeak, along with nonstationary, depth-dependent resolution compensation.

\section{Quality Control}

Reconstructed short-axis images were loaded into QPS. A senior, experienced nuclear cardiology core laboratory technologist who was unaware of all results, including the patient group studies (normal database, normalcy, and accuracy groups), examined the short-axis images and manually corrected them for proper left ventricular positioning; that is, short-axis cross hairs were positioned over the left ventricle center and long-axis line endpoints were positioned over the left ventricle apex and base, separately for AC and NC studies, when necessary. All corrections were made strictly following the contour correction specified in the QPS software manual. Corrections were made only for obvious discrepancies to avoid any unnecessary manipulation of the data. The process included insertion of the left ventricle center on axial, vertical long-axis, and horizontal long-axis images, followed by masking of the region outside the left ventricle in case of extracardiac activity. All other processing was fully automated to ensure maximal reliability of the data.

To minimize the chances of data collection errors, the laboratory acquiring the studies used a strict study regimen, which included meticulous attention to detail with regard to patient preparation, stress testing and injection, image acquisition, and reconstruction study protocols. In addition, we confined the manual intervention during the processing to the adjustment of the left ventricle boundaries, and this intervention was performed as infrequently as possible and in a masked fashion by the operator.

\section{Perfusion Quantification and Quantitative Software Analysis}

Quantitative analysis was performed with QPS (16). The method of quantification with QPS was previously described $(15,17,18)$. The following quantitative results were obtained for the AC and NC datasets: summed stress score (SSS), total perfusion deficit (TPD) (15), and regional defect extent for the left anterior descending artery (LAD), left circumflex artery (LCX), and right coronary artery (RCA).

For the direct comparison of quantification results, we used an SSS of $\geq 3$ to define the quantitative threshold for MPS abnormality (12) in a 17-segment model. However, because we recently proposed the TPD variable, which provides a more continuous measure of hypoperfusion (15), we also evaluated the use of that variable and obtained a precise TPD cutoff for the detection of abnormality for AC and NC studies with the LLk population. Assuming that LLk studies represent the perfusion pattern in the normal population, we defined the TPD value corresponding to the 95\% percentile in the LLk group as the optimal cutoff value for the detection of abnormality.

\section{Coronary Angiography}

Coronary angiography was performed with the standard Judkins approach, and all coronary angiograms were interpreted visually by experienced physicians. No patients had myocardial infarction or revascularization in the interval between MPS and coronary angiography. Two conventional cutoff points for CAD are regularly used in many studies: $\geq 70 \%$ - and $\geq 50 \%$-diameter stenosis. However, because the agreement between nuclear data and the angiographic cutoff point of $70 \%$ was found to be higher than that for $50 \%$, we chose to use the $\geq 70 \%$-diameter stenosis cutoff point (19); therefore, stenosis with a narrowing of $\geq 70 \%$ of the luminal diameter was considered significant. Coronary angiography findings are shown in Table 2.

\section{Statistical Analysis}

All continuous variables were expressed as mean \pm 1 SD. Paired $t$ tests were used to compare differences in paired continuous data, and McNemar tests were used to compare differences in paired discrete data. All statistical tests were 2-tailed, and a $P$ value of $<0.05$ was considered significant. For a comparison of regional patterns in studies with discrepant $\mathrm{AC}$ and $\mathrm{NC}$ results, a direct calculation of probability was performed. For agreement assessment, correlations and bias plots were calculated. A receiver operating characteristic (ROC) curve analysis was performed to evaluate the abilities of the $\mathrm{AC}$ and $\mathrm{NC}$ datasets to predict $\geq 70 \%$ stenoses of coronary arteries. ROC curves were created by use of all possible integer score values and a step of $0.1 \%$ for the defect extent values. Correlations, bias plots, and differences between ROC curve areas (area \pm SE) were compared by use of Version 1.71 of the Analyze-It statistical package from Analyze-It Software Ltd. The ROC curves were compared with the Hanley and

TABLE 2

Angiographic Characteristics for 114 Patients

\begin{tabular}{lc}
\hline \multicolumn{1}{c}{ Parameter } & No. (\%) of patients \\
\hline No stenosis* & $45(40)$ \\
\hline Single-vessel disease & $31(27)$ \\
Double-vessel disease & $23(20)$ \\
Triple-vessel disease & $15(13)$ \\
LAD disease & $55(48)$ \\
LCX disease & $46(40)$ \\
RCA disease & $51(45)$ \\
\hline
\end{tabular}

*Stenosis of $\geq 70 \%$ luminal diameter narrowing was considered significant. 
TABLE 3

Quantitative Parameters Obtained for AC and NC Data

\begin{tabular}{lccl}
\hline & \multicolumn{2}{c}{ Mean \pm SD for: } & \\
\cline { 2 - 3 } Parameter (no. of patients) & NC & AC & \\
\hline SSS-all (114) & $6.7 \pm 8.2$ & $6.9 \pm 8.0$ & 0.5 \\
\hline SSS-no CAD (45) & $1.6 \pm 2.3$ & $1.8 \pm 2.5$ & 0.6 \\
SSS-CAD (69) & $10.0 \pm 9.0$ & $10.2 \pm 8.5$ & 0.6 \\
SSS-LLk (134) & $0.5 \pm 1$ & $0.6 \pm 1.2$ & 0.23 \\
TPD-all (114) & $8.5 \pm 11.5$ & $9.4 \pm 11.7$ & 0.07 \\
TPD-no CAD (45) & $1.6 \pm 2.4$ & $2.1 \pm 2.6$ & 0.2 \\
TPD-CAD (69) & $12.9 \pm 12.9$ & $14.1 \pm 12.1$ & 0.1 \\
TPD-LLk (134) & $0.4 \pm 1.0$ & $0.7 \pm 1.1$ & 0.18 \\
\hline
\end{tabular}

McNeil method, which requires that all tests be performed on the same subjects (20).

\section{RESULTS}

\section{Quality Control}

The contours needed to be adjusted in 17 of 248 (7\%) and 25 of $248(10 \%)$ of the $\mathrm{NC}$ and $\mathrm{AC}$ stress studies, respectively $(P=0.1)$.

\section{Quantitative Results}

The mean SSS and mean TPD for the whole group and for subgroups are shown in Table 3. There were no differences in the mean SSS and mean TPD between NC and AC. Figure 1 shows correlations and bias plots (BlandAltman analysis) obtained with the 2 methods for SSS (left) and TPD (right). With both methods, there was only minor bias, and the correlations between $\mathrm{AC}$ and $\mathrm{NC}$ results were high for both SSS and TPD (0.89 and 0.90, respectively) $(P<0.0001)$. The normalcy, sensitivity, specificity, and accuracy obtained when the SSS threshold of $\geq 3$ was applied are shown in Figure 2. There were no statistically significant differences between the 2 methods. Figure 3 shows the ROC curves for the detection of $\geq 70 \%$ stenosis by SSS (Fig. 3A) and by TPD (Fig. 3B); as shown, the areas under the curves (AUCs) were similar with both methods and with both comparisons. Regional defect extent by coronary artery territory was also calculated and is shown in Figure 4. The ROC AUCs for CAD detection per vessel were similar with AC and NC. When we reanalyzed the data at $\mathrm{a} \geq 50 \%$ cutoff, similar results were found for accuracy, sensitivity, specificity, and ROC analysis. An additional analysis of diagnostic performance was done with the alternative method of defining the abnormality threshold for TPD. The 95\% percentiles for TPD were $2.7 \%$ and $2.8 \%$ for NC and $\mathrm{AC}$, respectively; therefore, for practical purposes, a TPD of $3 \%$, the nearest rounded integer value (as reported by the software to the user) for both NC and AC, was defined as the most optimal abnormality threshold. The sensitivity, specificity, and accuracy obtained when the TPD threshold of
FIGURE 1. Correlations and bias plots (Bland-Altman analysis) for SSS and TPD and for NC and AC.

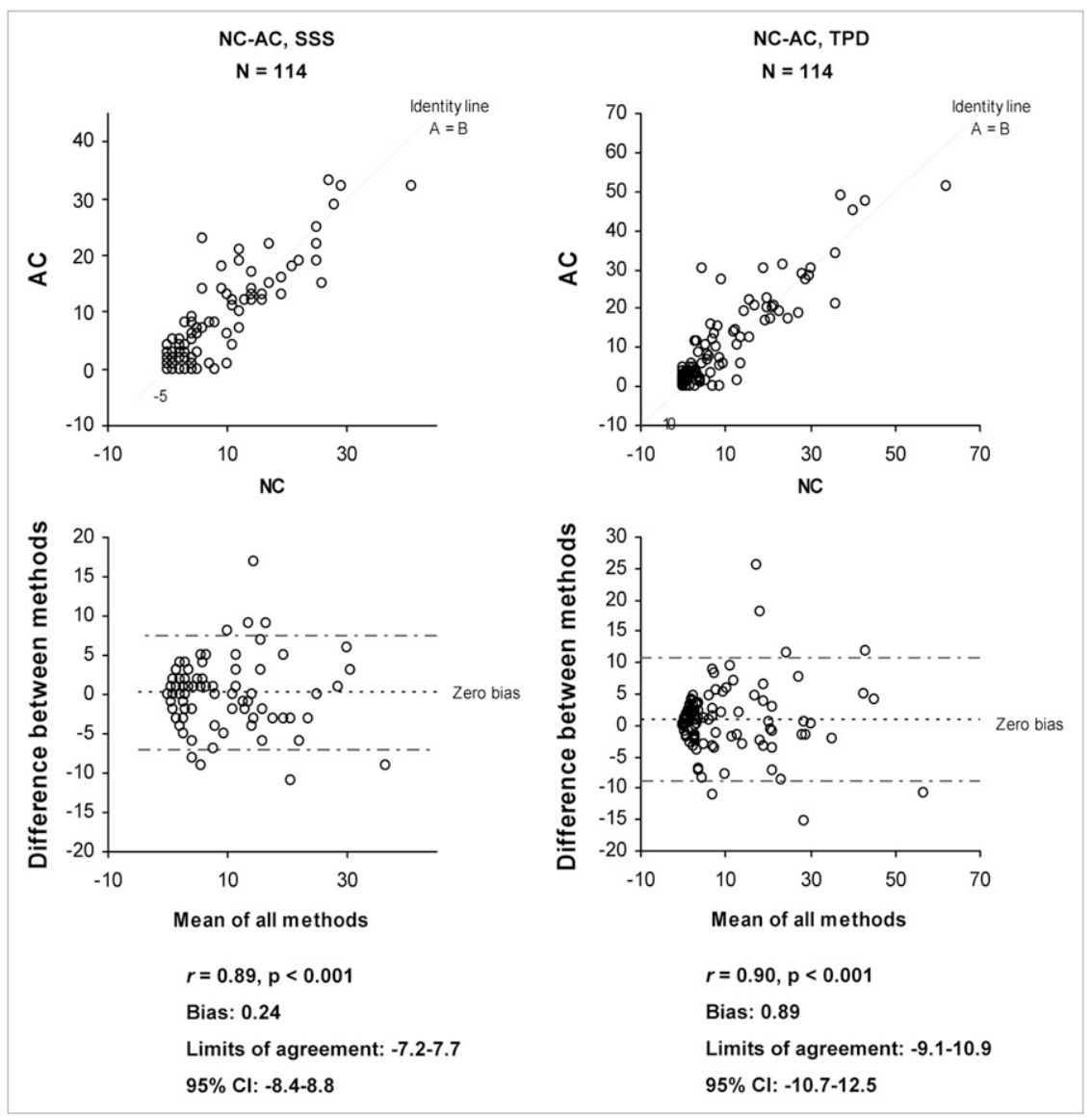




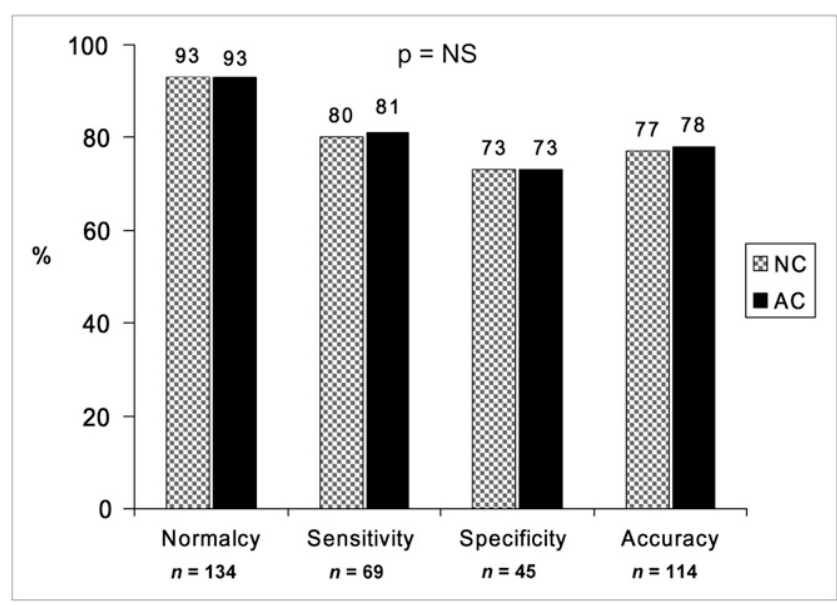

FIGURE 2. Comparison of quantitative results obtained with $\mathrm{NC}$ and $\mathrm{AC}$ for SSS abnormality threshold of $\geq 3$. NS $=$ not significant.

$\geq 3.0 \%$ was applied are shown in Figure 5. There were still no significant differences between the 2 methods, although the TPD analysis showed a trend for higher sensitivity with AC $(P=0.3)$.

We separately analyzed all of the discrepant cases (where $\mathrm{AC}$ and $\mathrm{NC}$ results differed) to understand the possible differences between $\mathrm{NC}$ quantification and $\mathrm{AC}$ quantification. In all 12 cases of false-positive AC results, there was an LAD perfusion defect. There were also one case with additional RCA and one case with additional LCX perfusion defects. In 9 of 12, 6 of 12, and 9 of 12 cases of falsepositive NC results, there were $\mathrm{LAD}, \mathrm{LCX}$, and RCA perfusion defects, respectively. In all 8 cases with falsepositive $\mathrm{AC}$ results and true-negative $\mathrm{NC}$ results, the falsepositive AC perfusion defect was in the LAD territory $(P<$ $0.01)$. On the other hand, in all 8 cases with false-positive $\mathrm{NC}$ results and true-negative $\mathrm{AC}$ results, the false-positive NC perfusion defect was in the RCA territory $(P<0.01)$. The quality of all 8 false-positive AC cases was good to excellent, and there was no motion or excessive extracardiac activity. Figure 6 shows examples for 3 cases.

\section{DISCUSSION}

To the best of our knowledge, this is the first study that compared (quantitative) attenuation-corrected data specifically in a female population. There was only one other study, by Gallowitsch et al., reporting the performance of AC separately in a limited population of females $(n=38)$, but assessment by visual techniques was used in that study (2). The quantitative analysis used in our study avoids any kind of bias that might be possible with visual analysis. Our results show that there is no measurable difference in the degree of automation and in the diagnostic performance when $\mathrm{AC}$ and $\mathrm{NC}$ data for women are compared.

The apparent lack of advantage of $\mathrm{AC}$ over $\mathrm{NC}$ in the diagnostic performance of quantification can have several explanations. The improved diagnostic performance of $\mathrm{AC}$ in the vast majority of previous studies was attributed mainly to improvement in the normalcy rate and specificity for detecting CAD. However, because of "AC-induced" apical thinning and truncation artifacts, $\mathrm{AC}$ might paradoxically create false-positive perfusion defects in a normal myocardium, thus subsequently reducing the specificity $(21,22)$. Visual analysis might be able to circumvent this problem, because all of the reported studies used both $\mathrm{NC}$ and $\mathrm{AC}$ data in the final visual analysis. Interestingly, the relative incidence of apical thinning was found to be more than twice as high for women as for men, 4.1 versus 1.9 (22), and therefore may represent a relatively larger problem in women than in the general population. Figure 6B shows an example of this problem in a patient with normal coronary arteries at angiography, a normal NC study, but apical thinning on an AC study, leading to a false-positive perfusion defect. Also, there is some evidence that the apparent effects of scatter, patient or organ motion, and variable resolution in noncircular orbits are more pronounced when AC is used (21), possibly influ-

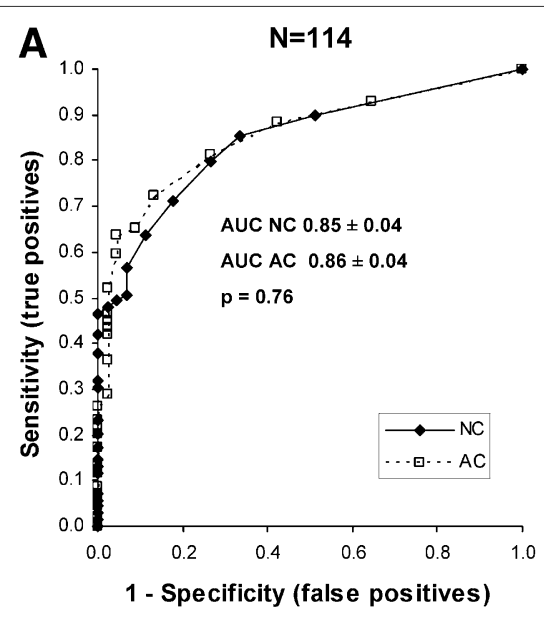

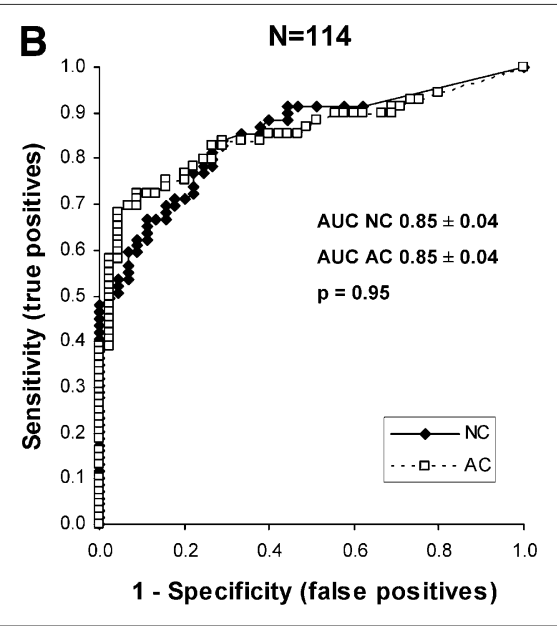

FIGURE 3. ROC curves for detection of $\geq 70 \%$ stenosis with SSS (A) and TPD (B). 
FIGURE 4. ROC curves for detection of $\geq 70 \%$ stenosis by coronary artery territory.

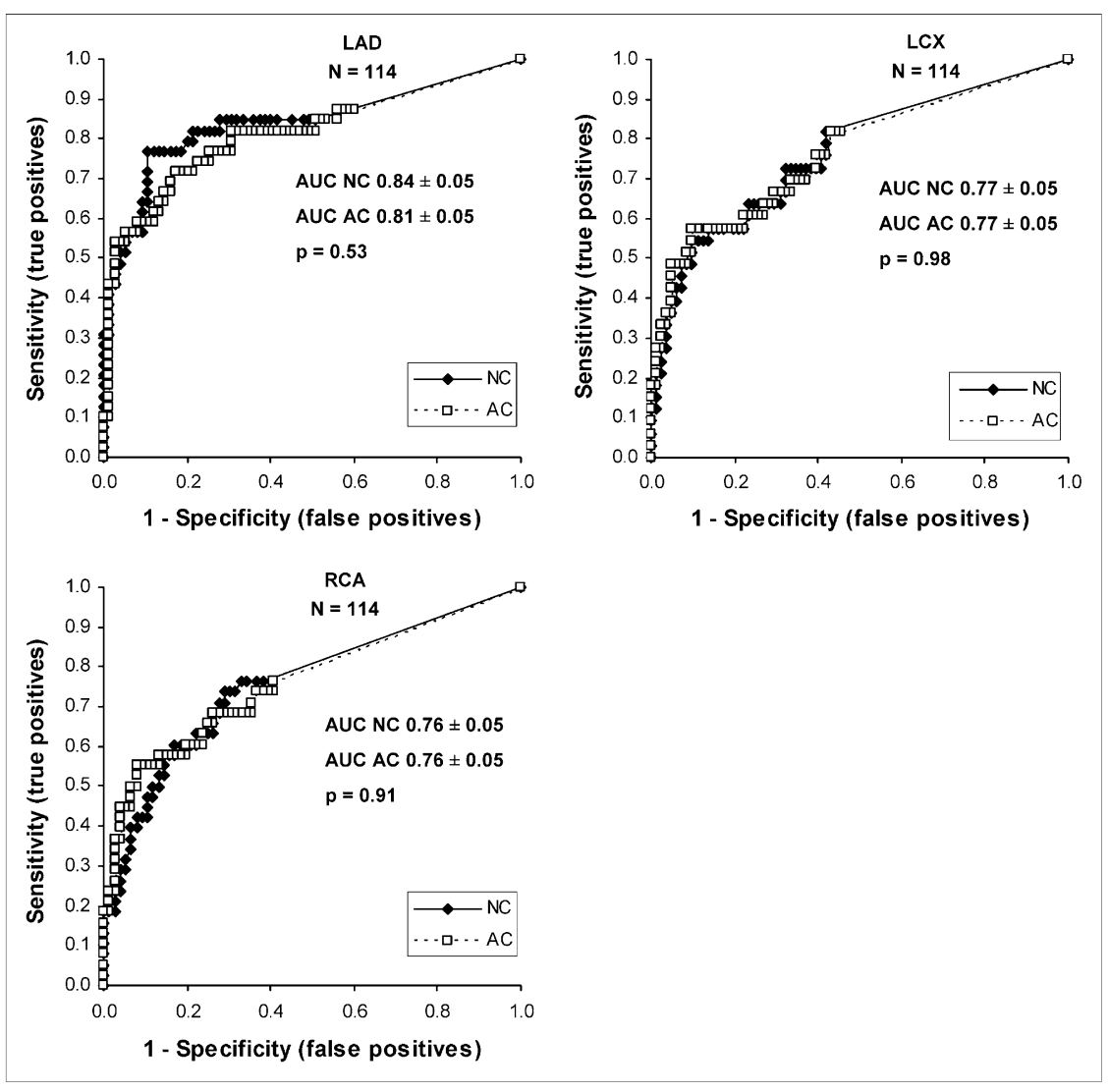

encing accuracy. Because, in the present study, the AC implementation included scatter correction and none of the AC false-positive cases demonstrated motion, the falsepositive findings cannot be attributed to the effects of scatter or motion.

Furthermore, the previously reported low specificity of most quantitative programs with NC data was often associated with older algorithms, known to be limited by low specificity (23). More recent algorithms developed by various groups achieved higher specificity but did not support the hypothesis of a significant measurable advantage of AC over NC (12,24). Therefore, it is possible that the incremental diagnostic benefit of AC is inversely related to the sophistication of the specific quantitative algorithm used to measure it and that, when a new-generation algorithm is used, as in the present study, NC performance is more comparable to $\mathrm{AC}$ performance.

The present study showed better detection of RCA territory false-positive lesions with $\mathrm{AC}$, yet the poorer performance in the LAD territory counterbalanced this result. It also showed that when the optimal TPD threshold for abnormality is used, there is a trend for higher sensitivity of $\mathrm{AC}$ than of $\mathrm{NC}$, as shown in Figure $6 \mathrm{C}$; this result is quite encouraging. Therefore, it is possible that refinements in $\mathrm{AC}$ to lower the LAD territory false-positive defects and the optimal TPD threshold will allow improved quantification of AC studies. Furthermore, because the discrepant AC and NC results evidently had different sources of errors (AC falsepositive results in the LAD territory and NC false-positive results mostly in the RCA territory), a potential performance gain could be accomplished with a combination of quantitative AC and NC analyses emulating a visual analysis similar to our supine-prone analysis (25) to fully realize the potential of AC imaging with quantitative software. To our knowledge,

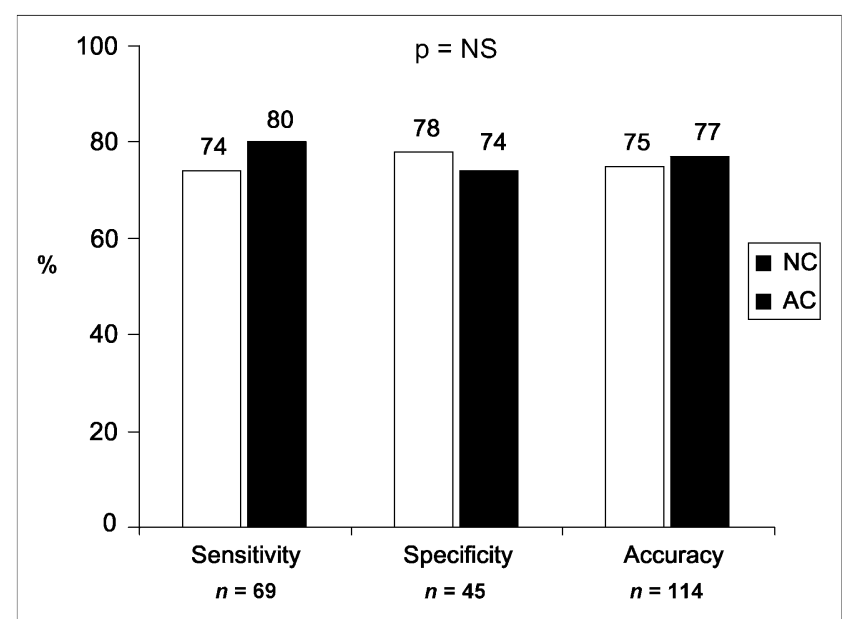

FIGURE 5. Comparison of quantitative results obtained with $\mathrm{NC}$ and $\mathrm{AC}$ for TPD abnormality threshold of $\geq 3$. NS $=$ not significant. 


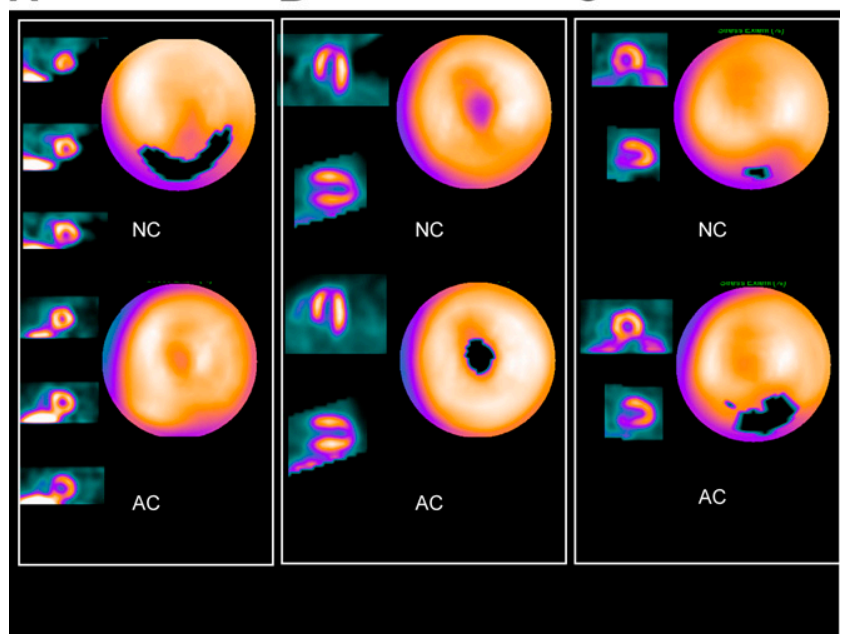

FIGURE 6. Examples for 3 cases. (A) 55-y-old hypertensive, obese $(\mathrm{BMI}=35)$ patient who had atypical chest pain and who underwent pharmacologic stress MPS. Coronary angiography revealed no evidence of CAD. NC image (top) revealed basal inferior perfusion defect in territory of RCA, whereas AC image (bottom) depicted normal study. (B) 67-y-old overweight (BMI = 26), hypertensive, dyslipidemic, diabetic patient who underwent exercise stress MPS. NC image (top) depicted normal study, whereas $A C$ image (bottom) revealed perfusion defect in territory of LAD. This perfusion defect might have been attributable to apical thinning apparent on AC but not NC studies. (C) 62-y-old hypertensive, diabetic, dyslipidemic, morbidly obese $(\mathrm{BMI}=40)$ patient who had atypical chest pain and who underwent pharmacologic stress MPS. Coronary angiography revealed 90\% RCA stenosis. NC image (top) revealed minimal nonsignificant perfusion defect. AC image (bottom), however, correctly revealed basal inferior perfusion defect in territory of RCA.

however, none of the existing quantification algorithms supports such an analysis.

The limitations of the present study are the use of angiography as a gold standard, which may not correspond to the physiologic significance of the defect, and the need for some manual corrections (although there were no differences in the rates of manual corrections for $\mathrm{AC}$ and $\mathrm{NC}$ ). Also, the present study was designed to compare $\mathrm{AC}$ and $\mathrm{NC}$ in a fully automated fashion without any visual interpretation. It is reasonable to assume that side-by-side interpretations of $\mathrm{AC}$ and NC studies will have additive favorable effects on overall accuracy, because, conceivably, in such a combined analysis, routinely observed false-positive defects such as apical thinning will be ignored. Of note, although we found that $\mathrm{AC}$ was associated with false-positive results in the LAD territory whereas NC was associated with false-positive results in the RCA territory, these results were obtained retrospectively. Because there may be differences among various $\mathrm{AC}$ methods, these results apply only to the data collection, reconstruction, and analysis described in this article. Additional studies may be warranted for other AC methods. Finally, the present study may not have had enough subjects to prove the advantage of AC, because a trend for higher sensitivity was noted but did not reach statistical significance; nevertheless, this is the largest study to date on the effects of $\mathrm{AC}$ in women.

\section{CONCLUSION}

There are no significant diagnostic differences between automatic quantitative MPS analyses performed with QPS in studies processed with and without AC in women. AC was associated with false-positive results in the LAD territory, whereas NC was associated with false-positive results in the RCA territory.

\section{ACKNOWLEDGMENTS}

The authors thank James Gerlach, CNMT, who examined and corrected the MPS contours. Arik Wolak is a Save A Heart Foundation, Inc., Fellow at Cedars-Sinai Medical Center, Los Angeles, CA, and APF, Boston, MA.

\section{REFERENCES}

1. Heller GV, Links J, Bateman TM, et al. American Society of Nuclear Cardiology and Society of Nuclear Medicine joint position statement: attenuation correction of myocardial perfusion SPECT scintigraphy. J Nucl Cardiol. 2004;11:229-230.

2. Gallowitsch HJ, Sykora J, Mikosch P, et al. Attenuation-corrected thallium-201 single-photon emission tomography using a gadolinium-153 moving line source: clinical value and the impact of attenuation correction on the extent and severity of perfusion abnormalities. Eur J Nucl Med. 1998;25:220-228.

3. Hendel RC, Berman DS, Cullom SJ, et al. Multicenter clinical trial to evaluate the efficacy of correction for photon attenuation and scatter in SPECT myocardial perfusion imaging. Circulation. 1999;99:2742-2749.

4. Links JM, Becker LC, Rigo P, et al. Combined corrections for attenuation, depthdependent blur, and motion in cardiac SPECT: a multicenter trial. $\mathrm{J} \mathrm{Nucl}$ Cardiol. 2000;7:414-425.

5. Links JM, DePuey EG, Taillefer R, Becker LC. Attenuation correction and gating synergistically improve the diagnostic accuracy of myocardial perfusion SPECT. J Nucl Cardiol. 2002;9:183-187.

6. Thompson RC, Heller GV, Johnson LL, et al. Value of attenuation correction on ECG-gated SPECT myocardial perfusion imaging related to body mass index. J Nucl Cardiol. 2005;12:195-202.

7. Masood Y, Liu YH, Depuey G, et al. Clinical validation of SPECT attenuation correction using $\mathrm{x}$-ray computed tomography-derived attenuation maps: multicenter clinical trial with angiographic correlation. J Nucl Cardiol. 2005;12:676686.

8. Ficaro EP, Fessler JA, Shreve PD, Kritzman JN, Rose PA, Corbett JR. Simultaneous transmission/emission myocardial perfusion tomography: diagnostic accuracy of attenuation-corrected ${ }^{99 \mathrm{~m}} \mathrm{Tc}$-sestamibi single-photon emission computed tomography. Circulation. 1996;93:463-473.

9. Kluge R, Sattler B, Seese A, Knapp WH. Attenuation correction by simultaneous emission-transmission myocardial single-photon emission tomography using a technetium-99m-labelled radiotracer: impact on diagnostic accuracy. Eur J Nucl Med. 1997;24:1107-1114.

10. Grossman GB, Garcia EV, Bateman TM, et al. Quantitative Tc-99m sestamibi attenuation-corrected SPECT: development and multicenter trial validation of myocardial perfusion stress gender-independent normal database in an obese population. J Nucl Cardiol. 2004;11:263-272.

11. Shotwell M, Singh BM, Fortman C, Bauman BD, Lukes J, Gerson MC. Improved coronary disease detection with quantitative attenuation-corrected Tl-201 images. J Nucl Cardiol. 2002;9:52-62.

12. Slomka PJ, Fish MB, Lorenzo S, et al. Simplified normal limits and automated quantitative assessment for attenuation-corrected myocardial perfusion SPECT. J Nucl Cardiol. 2006;13:642-651.

13. Hendel RC. Attenuation correction: eternal dilemma or real improvement? $Q J$ Nucl Med Mol Imaging. 2005;49:30-42.

14. Diamond GA, Forrester JS. Analysis of probability as an aid in the clinical diagnosis of coronary-artery disease. N Engl J Med. 1979;300:1350-1358.

15. Slomka PJ, Nishina H, Berman DS, et al. Automated quantification of myocardial perfusion SPECT using simplified normal limits. J Nucl Cardiol. 2005;12: $66-77$. 
16. Germano G, Gerlach J. QPS \& ARG: Generic User Manual. Los Angeles, CA: Artificial Intelligence in Medicine Program, Department of Medicine, CedarsSinai Medical Center; 2004. QPS-UM-GENERIC-001-rev3-02NL.

17. Germano G, Kavanagh PB, Waechter P, et al. A new algorithm for the quantitation of myocardial perfusion SPECT. I. Technical principles and reproducibility. J Nucl Med. 2000;41:712-719.

18. Sharir T, Germano G, Waechter PB, et al. A new algorithm for the quantitation of myocardial perfusion SPECT. II. Validation and diagnostic yield. J Nucl Med. 2000;41:720-727.

19. Heller LI, Cates C, Popma J, et al. Intracoronary Doppler assessment of moderate coronary artery disease: comparison with ${ }^{201} \mathrm{Tl}$ imaging and coronary angiography. Circulation. 1997;96:484-490.

20. Hanley JA, McNeil BJ. A method of comparing the areas under receiver operating characteristic curves derived from the same cases. Radiology. 1983; 148:839-843.
21. Case J, Cullom S, Bateman TM. Myocardial Perfusion Single-Photon Emission Computed Tomography Attenuation Correction. 3rd ed. New York, NY: Oxford University Press; 2003.

22. Links JM, Becker LC, Anstett F. Clinical significance of apical thinning after attenuation correction. J Nucl Cardiol. 2003;11:26-31.

23. Van Train KF, Garcia EV, Maddahi J, et al. Multicenter trial validation for quantitative analysis of same-day rest-stress technetium- $99 \mathrm{~m}$-sestamibi myocardial tomograms. J Nucl Med. 1994;35:609-618.

24. Wolak A, Slomka PJ, Fish MB, et al. Automated analysis of attenuation-corrected SPECT: comparison of two state-of-the-art software packages [abstract]. $\mathrm{J} \mathrm{Nucl}$ Cardiol. 2007;14(suppl):S110.

25. Nishina H, Slomka PJ, Abidov A, et al. Combined supine and prone quantitative myocardial perfusion SPECT: method development and clinical validation in patients with no known coronary artery disease. J Nucl Med. 2006;47: 51-58. 